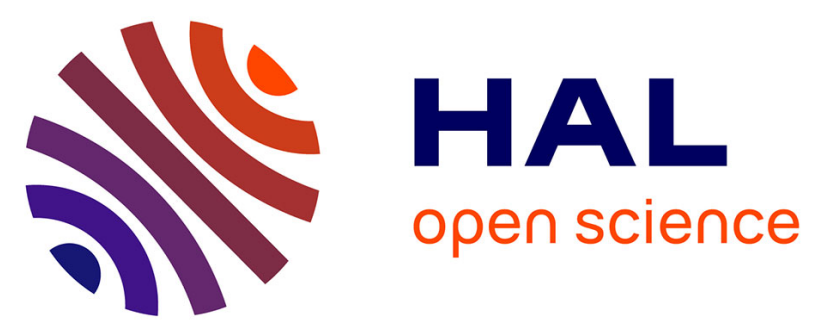

\title{
Bifurcation analysis versus maximum force criteria in formability limit assessment of stretched metal sheets
}

Farid Abed-Meraim, Ron H. J. Peerlings, Marc G.D. Geers

\section{To cite this version:}

Farid Abed-Meraim, Ron H. J. Peerlings, Marc G.D. Geers. Bifurcation analysis versus maximum force criteria in formability limit assessment of stretched metal sheets. International Journal of Applied Mechanics, 2014, 6 (6), pp.1450064. 10.1142/S1758825114500641 . hal-01199864

\section{HAL Id: hal-01199864 \\ https://hal.science/hal-01199864}

Submitted on 16 Sep 2015

HAL is a multi-disciplinary open access archive for the deposit and dissemination of scientific research documents, whether they are published or not. The documents may come from teaching and research institutions in France or abroad, or from public or private research centers.
L'archive ouverte pluridisciplinaire HAL, est destinée au dépôt et à la diffusion de documents scientifiques de niveau recherche, publiés ou non, émanant des établissements d'enseignement et de recherche français ou étrangers, des laboratoires publics ou privés. 


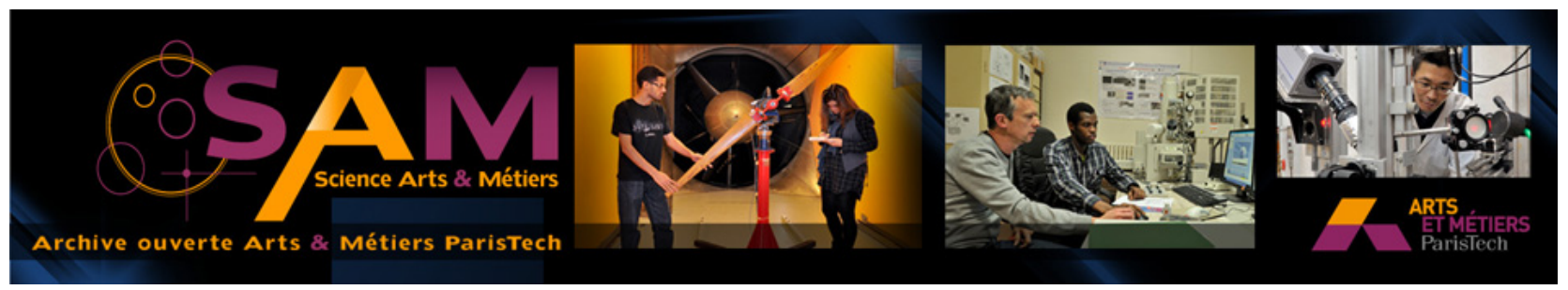

\section{Science Arts \& Métiers (SAM)}

is an open access repository that collects the work of Arts et Métiers ParisTech researchers and makes it freely available over the web where possible.

This is an author-deposited version published in: http://sam.ensam.eu

Handle ID: .http://hdl.handle.net/10985/10023

\section{To cite this version :}

Farid ABED-MERAIM, Ron H. J. PEERLINGS, Marc G.D. GEERS - Bifurcation analysis versus maximum force criteria in formability limit assessment of stretched metal sheets - International Journal of Applied Mechanics - Vol. 6, n6, p.27 p. - 2014 


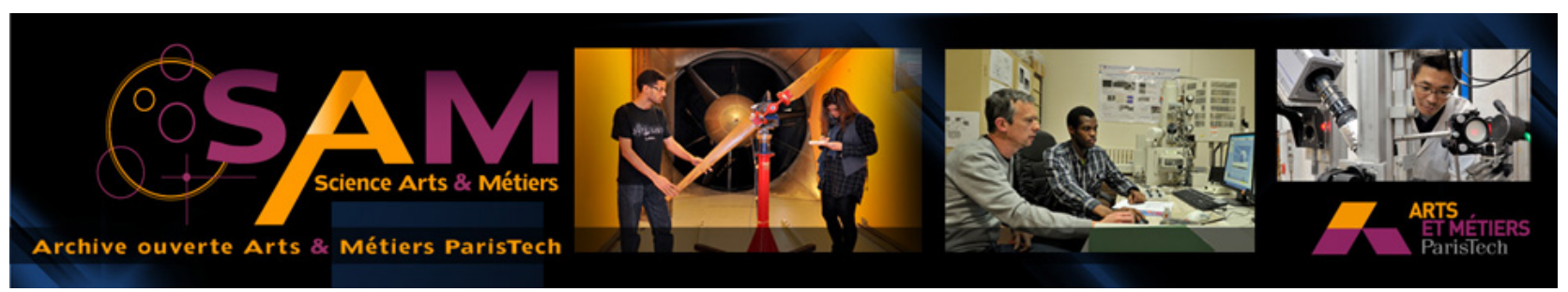

\section{Science Arts \& Métiers (SAM)}

is an open access repository that collects the work of Arts et Métiers ParisTech researchers and makes it freely available over the web where possible.

This is an author-deposited version published in: http://sam.ensam.eu

Handle ID: .http://hdl.handle.net/null

\section{To cite this version :}

Farid ABED-MERAIM, Ron H.J. PEERLINGS, Marc G.D. GEERS - Bifurcation analysis versus maximum force criteria in formability limit assessment of stretched metal sheets - Bifurcation analysis versus maximum force criteria in formability limit assessment of stretched metal sheets Vol. $6, n^{\circ} 6$, p.27 p- 2014 


\title{
BIFURCATION ANALYSIS VERSUS MAXIMUM FORCE CRITERIA IN FORMABILITY LIMIT ASSESSMENT OF STRETCHED METAL SHEETS
}

\author{
F. ABED-MERAIM* ${ }^{*}$, R. H. J. PEERLINGS ${ }^{\dagger}$ and M. G. D. GEERS ${ }^{\dagger}$ \\ *Laboratoire d'Étude des Microstructures et de Mécanique des Matériaux \\ LEM3, UMR CNRS 7239, Arts et Métiers ParisTech \\ 4 rue Augustin Fresnel, 57078 Metz Cedex 3, France \\ ${ }^{\dagger}$ Department of Mechanical Engineering \\ Eindhoven University of Technology, P.O. Box 513 \\ 5600 MB Eindhoven, The Netherlands \\ ¥farid.abed-meraim@ensam.eu
}

\begin{abstract}
The present contribution deals with the prediction of diffuse necking in the context of forming and stretching of metal sheets. For this purpose, two approaches are investigated, namely bifurcation and the maximum force principle, with a systematic comparison of their respective ability to predict necking. While the bifurcation approach is of quite general applicability, some restrictions are shown for the application of maximum force conditions. Although the predictions of the two approaches are identical for particular loading paths and constitutive models, they are generally different, which is even the case for elasticity, confirming the distinct nature of the two concepts. Closed-form expressions of the critical stress and strain states are derived for both criteria in elasto-plasticity and rigid-plasticity for a variety of hardening models. The resulting useful formulas in rigidplasticity are shown to also accurately represent the elasto-plastic critical states for small ratios of the hardening modulus with respect to Young's modulus. Finally, the well-known expression of Swift's diffuse necking criterion, whose foundations are attributed in the literature to the maximum force principle, is shown here to originate from the bifurcation approach instead, providing a sound justification for it.
\end{abstract}

Keywords: Bifurcation; maximum force principle; forming limits; diffuse and localized necking; stretched metal sheets; formability.

\section{Introduction}

In the literature dealing with plastic instabilities, and the analysis of sheet metal forming processes, quite a large number of forming limit criteria have been developed. However, a comprehensive and rigorous comparison of their theoretical foundations and underlying assumptions is seldom made. Listing all these criteria proves 
to be difficult, considering the multitude of variants derived from some of these approaches. A short review reveals, however, that those criteria could be classified into distinct categories, depending on their fundamental basis as well as their theoretical or physical background.

For stretched metal sheets, two forms of necking may occur, namely diffuse and localized necking. It has been shown that diffuse necking appears prior to localized necking, and it is now well recognized that the maximum allowable strain in sheet metal forming is restricted by localized necking. For this reason, most of the theoretical Forming Limit Diagrams (FLDs) are now based on the onset of localization. From an experimental viewpoint, the FLDs are obtained at localized necking for different loading paths (uniaxial tensile (UT) test, biaxial tensile tests, plane-strain tensile (PT) test) using Marciniak's or Nakazima's test with specimens of different widths. Note that this FLD concept was first introduced by Keeler [1965] and Goodwin [1968] in order to determine the critical strains that could lead to defective parts.

Early instability criteria were based on the maximum force principle, originating from Considère [1885] and its two-dimensional (2D) extension by Swift [1952] for application to metal sheets. These criteria, in their original form, were known to predict diffuse necking. Later, these maximum-force-based criteria were extended by Hora et al. [1996] and subsequently by Mattiasson et al. [2006] in order to predict localized necking, and enhanced versions were developed to take into account thickness effects, strain-path changes, etc. Note also that Hill's zero-extension criterion [Hill, 1952], predicting localized necking on the left-hand side of the FLD, was developed almost in the same period as Swift's diffuse necking criterion.

Another approach, postulating a pre-existing defect in the material sheet, was proposed by Marciniak and Kuczynski [1967]. In its original version, the MarciniakKuczynski $(\mathrm{M}-\mathrm{K})$ model can be regarded, in a sense, as a complementary approach to Hill's zero-extension criterion, since no zero-extension direction exists for positive biaxial stretching. However, since localized necking in biaxial stretching is observed in practice, a geometric imperfection has to be introduced in the $\mathrm{M}-\mathrm{K}$ model to capture this phenomenon, which may provide some justification to this imperfection theory. This model was subsequently extended by Hutchinson and Neale [1978] to the prediction of the left-hand side of the FLD by allowing the imperfection band to rotate until a localized neck is detected.

Drucker's and Hill's theory of loss of material stability [Drucker, 1956; Hill, 1958], also referred to as the general bifurcation criterion, represents another class of approaches for necking prediction. Belonging to the same class, limit-point bifurcation appeared later [Valanis, 1989], and it has been shown that for associative elasto-plasticity, limit-point bifurcation coincides with general bifurcation. For localized necking, Rudnicki and Rice established a bifurcation criterion based on loss of ellipticity (i.e., singularity of the acoustic tensor), also known as the discontinuous bifurcation criterion [Rudnicki and Rice, 1975; Rice, 1976]. In the same way, some 
authors suggested the use of loss of strong ellipticity [Bigoni and Hueckel, 1991; Neilsen and Schreyer, 1993], which was shown to coincide with Rice's criterion for associative elasto-plastic models.

A final significant class of criteria concerns those based on stability theory. Within this approach, necking and localization phenomena are tackled by stability analysis of the local equilibrium equations. The starting point is the mathematical concept of stability, introduced by Lyapunov [1892] and commonly applied to structural instability problems [see, e.g., Abed-Meraim, 1999; Mojahedi et al., 2013]. The associated technique of linear perturbation was extended to material instability problems by Molinari and Clifton [1987]. To investigate the rate of growth of the perturbation, its governing equations are linearized, and the resulting eigenvalue problem will characterize stable and unstable modes. For strain-rate dependent material models, this approach could be regarded as an interesting alternative to bifurcation theory; the latter is known not to apply for strain-rate sensitive materials. In the limit of vanishing viscous effects, this methodology was shown to recover Rice's bifurcation criterion [Barbier et al., 1998; Benallal, 2008].

From the above overview of the various approaches for necking and localization prediction, an interesting observation can be made. Indeed, while the $\mathrm{M}-\mathrm{K}$ analysis and Maximum Force Criteria (MFC) have been widely used in the literature, few applications of Rice's strain localization theory to sheet metal formability have been published, and they are mostly restricted to plane-stress assumptions and simple constitutive models [see, e.g., Doghri and Billardon, 1995]. Recently, Rice's bifurcation criterion has been used to investigate formability limits of metallic materials (see, Haddag et al. [2009] and Mansouri et al. [2014] using phenomenological constitutive modeling and Franz et al. [2009] using micromechanical approaches). Besides its sound theoretical basis, this bifurcation criterion has also been shown to provide a useful tool to investigate the impact of microstructural mechanisms on the formability limit of multiphase polycrystalline materials [Franz et al., 2011; 2013].

In the present contribution, attention is restricted to diffuse necking predictions applied to the in-plane stretching of metal sheets. Although the investigation of diffuse necking, as an approach to formability limits, may be seen as conservative when compared to localized necking predictions, there is yet a need for the former analysis. Besides its evident academic interest, explicit expressions for the critical hardening moduli allow loading paths, which are less favorable to necking, to be selected, and can therefore be used in experiments specifically designed for the material parameter identification relying on mechanical tests with homogeneous deformation. To this end, two approaches are thoroughly investigated, i.e., the maximum force principle and the bifurcation approach. Their respective ability to predict this type of geometric instability phenomena are systematically compared for different constitutive models.

The paper is outlined as follows. In Sec. 2, the problem statement is given along with the basic equations that govern the bifurcation analysis and the maximum 
force principle. Some restrictions regarding the range of validity of the MFC are also pointed out and discussed. Section 3 is a preliminary illustrative study within the framework of elasticity, where the predictions of the two approaches are shown to differ significantly, which confirms that they are based on distinct concepts. In Sec. 4, the critical stress and strain states are derived within the framework of elasto-plasticity for both criteria as closed-form expressions valid for a variety of hardening models. The analysis is then specialized in Sec. 5 for rigid-plasticity, where the resulting formulas are shown to accurately represent the elasto-plastic critical states for small ratios of the hardening modulus with respect to Young's modulus. Finally, some concluding remarks are given in Sec. 6. For completeness and practical applications, the critical hardening moduli and the associated critical strains are provided in Appendix A for a set of popular hardening laws.

\section{Equilibrium Equations and Problem Statement}

In this section, the problem statement and some general considerations will be given. Because some equations and principles are of quite general validity and common to all of the remaining sections of the paper, it has been chosen to summarize them in the current section for conciseness. These include the equilibrium equations, which are independent of the selected constitutive equations, the governing equations underlying the bifurcation analysis, and those related to the maximum force principle.

\subsection{Quasi-static equilibrium equations}

Let us consider a metal sheet as sketched in Fig. 1, which is subjected to biaxial loading conditions. The problem geometry is defined by its current parameters $l_{1}, l_{2}$, $l_{3}$ and their initial values $L_{1}, L_{2}, L_{3}$, which denote the dimensions along the major strain direction, the minor strain direction and the thickness direction, respectively.

The sheet is stretched by applying a biaxial loading state $\left(F_{1}(\lambda), F_{2}(\lambda)\right)$, in which $\lambda(t)$ is a monotonously increasing function acting as a load control parameter. The principal logarithmic strains, which remain parallel to the loading directions $(1,2)$

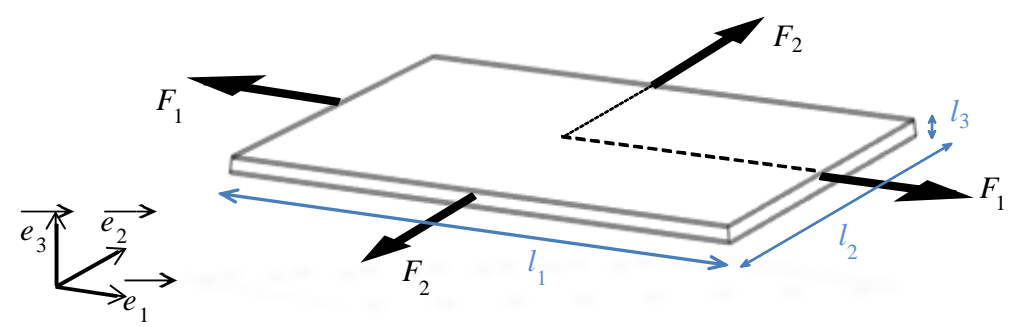

Fig. 1. Illustration of a metal sheet subjected to in-plane biaxial loading. 
(i.e., only isotropic material models are considered in this work), are given by

$$
\varepsilon_{i}=\ln \left(\frac{l_{i}}{L_{i}}\right), \quad i=1,2,3 .
$$

Using the principal Cauchy stress components $\left(\sigma_{1}, \sigma_{2}\right)$, the quasi-static equilibrium equations read

$$
\left\{\begin{array}{l}
F_{1}=\sigma_{1} l_{2} l_{3} \\
F_{2}=\sigma_{2} l_{1} l_{3}
\end{array}\right.
$$

which, combined with Eq. (1), can be rewritten as

$$
\left\{\begin{array}{l}
\frac{F_{1}}{L_{2} L_{3}}=\sigma_{1} e^{\left(\varepsilon_{2}+\varepsilon_{3}\right)} \\
\frac{F_{2}}{L_{1} L_{3}}=\sigma_{2} e^{\left(\varepsilon_{1}+\varepsilon_{3}\right)} .
\end{array}\right.
$$

For bifurcation analysis or application of MFC, the rate form of the equilibrium equations is usually more convenient, which reads

$$
\left\{\begin{array}{l}
\frac{\dot{F}_{1}}{l_{2} l_{3}}=\dot{\sigma}_{1}+\sigma_{1}\left(\dot{\varepsilon}_{2}+\dot{\varepsilon}_{3}\right) \\
\frac{\dot{F}_{2}}{l_{1} l_{3}}=\dot{\sigma}_{2}+\sigma_{2}\left(\dot{\varepsilon}_{1}+\dot{\varepsilon}_{3}\right) .
\end{array}\right.
$$

Note that so far, no constitutive equations had to be specified, since the above equilibrium equations do not depend on any material behavior law. However, these equations are often simplified, e.g., whenever the volume change can be neglected; this will be discussed at the end of this section.

\subsection{General bifurcation analysis}

The application of a biaxial loading state $\left(F_{1}(\lambda), F_{2}(\lambda)\right)$, which is characterized by the loading control parameter $\lambda(t)$, results in a quasi-static response indicated by superscript ${ }^{0}$. This response $\left(\varepsilon_{i}^{0}(\lambda), \sigma_{i}^{0}(\lambda)\right)$, referred to as the fundamental equilibrium path, may exhibit bifurcation when the loading reaches a critical value. Theoretically, this means that the equilibrium equations may lose uniqueness for some critical values of the loading. The bifurcated solution, which intersects the fundamental equilibrium path, is characterized by the critical load and the associated bifurcation mode. This type of instability phenomenon has been widely investigated in elasticity [see, e.g., Koiter, 1945; Timoshenko and Gere, 1961; Budiansky, 1974] as well as in plasticity [see, Hill, 1958; Hutchinson, 1974; Abed-Meraim and Nguyen, 2007]. For practical applications, the analysis of such buckling instabilities amounts to solving an eigenvalue problem, in which one seeks the first eigenvalue, corresponding to the lowest critical load, and the associated eigenmode [see, e.g., Liu et al., 2011; Gulshan Taj and Chakrabarti, 2013; Toh et al., 2013]. 
The bifurcation equations are classically obtained by first assuming that there exist two different solutions for the rate equilibrium equations (i.e., the fundamental path and the bifurcated solution). Then, the corresponding governing equations (i.e., the rate equilibrium equations associated with these two solutions) are subtracted from each other when evaluated at the first bifurcation point. At this bifurcation point, where the two solutions intersect, there may be only loss of uniqueness for some rate variables; the non-incremental quantities themselves are equal at this point of onset of bifurcation. Applying this procedure to Eq. (4), which represents the rate equilibrium equations for the above sheet under stretching, the bifurcation equations are derived as follows:

$$
\left\{\begin{array}{l}
\Delta \dot{\sigma}_{1}+\sigma_{1}^{0}\left(\Delta \dot{\varepsilon}_{2}+\Delta \dot{\varepsilon}_{3}\right)=0 \\
\Delta \dot{\sigma}_{2}+\sigma_{2}^{0}\left(\Delta \dot{\varepsilon}_{1}+\Delta \dot{\varepsilon}_{3}\right)=0
\end{array}\right.
$$

where $\Delta \dot{A}=\dot{A}-\dot{A}^{0}$ denotes the difference between any rate variable $\dot{A}$ evaluated on the bifurcated path and on the fundamental equilibrium path, respectively.

To proceed further with the bifurcation analysis (5), the constitutive equations are required, so that the corresponding eigenvalue problem is completely defined and can therefore be solved. This will be done in the subsequent sections, in which the superscript ${ }^{0}$ will be omitted for clarity.

\subsection{Maximum force principle}

This classical approach was coined by Considère [1885] who observed that necking occurs in a rounded bar under uniaxial tension when the applied load reaches a maximum. This earlier one-dimensional (1D) necking criterion has subsequently been extended to biaxial loading conditions [see Swift, 1952]. Note that both the Considère and Swift' 52 criteria are known to predict diffuse necking, which generally occurs prior to localized necking in the context of sheet metal forming. For the prediction of localized necking, Hill [1952] proposed a criterion based on the formation of a band of stationary extension, while Hora et al. [1996] extended Considère's criterion by taking into account the strain-path evolution after diffuse necking towards a PT state. It is worth noting that this class of criteria, referred to as the MFC, is noticeably popular in the literature and has been widely applied to determine FLDs at diffuse or localized necking.

In this section, attention is restricted to diffuse necking predictions by reconsidering the basic equations on which the Considère and Swift' 52 criteria are based. For the above-described sheet under stretching, the condition of maximum load along the major strain direction is given by

$$
\dot{\sigma}_{1}+\sigma_{1}\left(\dot{\varepsilon}_{2}+\dot{\varepsilon}_{3}\right)=0
$$

while the maximum force condition for the load parallel to the minor strain direction is

$$
\dot{\sigma}_{2}+\sigma_{2}\left(\dot{\varepsilon}_{1}+\dot{\varepsilon}_{3}\right)=0
$$


In the context of plasticity, the MFC have been mainly developed under the assumptions of incompressibility and a plane-stress state, which are well-justified within the biaxial loading conditions and large plastic deformations to which metal sheets are subjected. For instance, under these conditions, Eq. (6) leads to the well-known $1 \mathrm{D}$ expression of Considère's criterion (i.e., $\dot{\sigma}_{1} / \dot{\varepsilon}_{1}=d \sigma_{1} / d \varepsilon_{1}=\sigma_{1}$ ), which expresses that necking starts when the uniaxial hardening modulus $h=d \sigma_{1} / d \varepsilon_{1}$ drops to the value of the stress.

For biaxial loading, the extension by Swift [1952] suggests the application of the MFC at a simultaneous maximum of the forces. However, the simultaneous occurrence of maximum forces is only possible for some trivial loading paths. This has been demonstrated through experiments [see, e.g., Habbad, 1994], but can also be shown by further analyzing Eqs. (6) and (7). For the prediction of FLDs, a proportional loading is usually adopted, characterized by a constant parameter $\beta=\dot{\varepsilon}_{2} / \dot{\varepsilon}_{1}$ that defines the loading path. This also results in a linear relationship between the in-plane principal stress components (i.e., $\sigma_{2} / \sigma_{1}=\alpha$ ). In this case, it is straightforward from Eqs. (6) and (7) that a simultaneous maximum of the forces is only possible for $\alpha=0$ or $\beta=1$. The first case, $\alpha=0$, corresponds to the UT test, and the second, $\beta=1$, to equibiaxial expansion (EBE). Note that for both cases, the simultaneous maximum amounts to the condition of a maximum of the force along the major strain direction. Indeed, in UT the second condition (Eq. (7)) is obviously always satisfied, since $F_{2}=0 \Rightarrow \sigma_{2}=0$. For EBE, the symmetry of the problem reveals that condition (7) simply reduces to condition (6).

To summarize, it has been shown that the condition of a simultaneous maximum of the forces (Eqs. (6) and (7)) only occurs for two particular loading paths, whereas the condition of maximum force along the major strain direction (Eq. (6)) may be possible for the whole range of loading paths that make up an FLD, and could therefore represent an alternative criterion. On the other hand, the condition of maximum force along the minor strain direction (Eq. (7)) is shown not to hold for some loading paths; therefore, this latter condition will no longer be investigated in the subsequent sections.

\subsection{Incompressibility}

In what follows, for the sake of simplicity, the assumption of incompressibility will be adopted. This assumption is justified by three main motivations:

- In the framework of elasticity, which represents the preliminary study of Sec. 3, our main objective is to clarify some general aspects, and especially to emphasize that the bifurcation and maximum force principle are two distinct concepts.

- In elasto-plasticity, the deformation levels corresponding to the investigated phenomena (bifurcation, necking, etc.) are sufficiently large to justify neglecting the elastic strain, or at least its compressible part, as compared to the plastic strain. 
- It will allow a consistent comparison with results provided in the literature for the MFC, which are mostly available as closed-form expressions derived under the incompressibility assumption (i.e., rigid-plasticity).

Making use of volume conservation, the equilibrium Eq. (3) simplify to

$$
\left\{\begin{array}{c}
\frac{F_{1}}{L_{2} L_{3}}=\sigma_{1} e^{-\varepsilon_{1}} \\
\frac{F_{2}}{L_{1} L_{3}}=\sigma_{2} e^{-\varepsilon_{2}}
\end{array}\right.
$$

with their rate form given by:

$$
\left\{\begin{array}{l}
\frac{\dot{F}_{1}}{l_{2} l_{3}}=\dot{\sigma}_{1}-\sigma_{1} \dot{\varepsilon}_{1} \\
\frac{\dot{F}_{2}}{l_{1} l_{3}}=\dot{\sigma}_{2}-\sigma_{2} \dot{\varepsilon}_{2} .
\end{array}\right.
$$

With these simplified equilibrium equations, the preceding equations that govern bifurcation and maximum force conditions, Eq. (5) and Eqs. (6) and (7), respectively, can be easily modified accordingly.

\section{Preliminary Study in Elasticity}

The aim of this section is to illustrate the above-discussed concepts of bifurcation and maximum force principle in the simple case of linear elasticity. This preliminary study, allowing analytical solutions and closed-form expressions of the critical loads, constitutes a useful stepping stone towards more realistic constitutive models.

\subsection{Elastic fundamental equilibrium path}

Let us consider again the sheet under biaxial stretching shown in Fig. 1. The associated strain and stress tensors $\varepsilon$ and $\boldsymbol{\sigma}$ are given by their corresponding matrices $\boldsymbol{\varepsilon}$ and $\boldsymbol{\sigma}$ with respect to the Cartesian basis considered as

$$
\varepsilon=\left(\begin{array}{ccc}
\varepsilon_{1} & 0 & 0 \\
0 & \varepsilon_{2} & 0 \\
0 & 0 & -\left(\varepsilon_{1}+\varepsilon_{2}\right)
\end{array}\right), \quad \sigma=\left(\begin{array}{ccc}
\sigma_{1} & 0 & 0 \\
0 & \sigma_{2} & 0 \\
0 & 0 & 0
\end{array}\right)
$$

The governing constitutive equations for linear incompressible elasticity are given in tensorial form by

$$
\boldsymbol{\varepsilon}=\frac{3}{2 E} \boldsymbol{\sigma}-\frac{1}{2 E} \operatorname{tr}(\boldsymbol{\sigma}) \mathbf{I}_{2},
$$

where $E$ is the Young's modulus, $\operatorname{tr}()$ stands for the trace operator, and $\mathbf{I}_{2}$ denotes the second-order identity tensor. These constitutive equations can be rewritten in 
component form as

$$
\left\{\begin{array}{l}
\varepsilon_{1}=\frac{1}{2 E}\left(2 \sigma_{1}-\sigma_{2}\right) \\
\varepsilon_{2}=\frac{1}{2 E}\left(2 \sigma_{2}-\sigma_{1}\right)
\end{array}\right.
$$

or alternatively in terms of stress components:

$$
\left\{\begin{array}{l}
\sigma_{1}=\frac{2 E}{3}\left(2 \varepsilon_{1}+\varepsilon_{2}\right) \\
\sigma_{2}=\frac{2 E}{3}\left(2 \varepsilon_{2}+\varepsilon_{1}\right) .
\end{array}\right.
$$

By setting $\beta=\varepsilon_{2} / \varepsilon_{1}$, a constant parameter that characterizes the loading path ( $\beta \in[-1 / 2,1]$, e.g., $\beta=-1 / 2$ for $\mathrm{UT}, \beta=0$ for $\mathrm{PT}, \beta=1$ for $\mathrm{EBE})$, it can be shown that if $\sigma_{2} / \sigma_{1}=\alpha$, the following relationship between $\beta$ and $\alpha$ holds:

$$
\beta=\frac{2 \alpha-1}{2-\alpha} \Leftrightarrow \alpha=\frac{1+2 \beta}{2+\beta} .
$$

Combining the above equations, the fundamental equilibrium solution can be obtained as follows:

$$
\varepsilon^{0}(\lambda)=\lambda(t)\left(\begin{array}{ccc}
1 & 0 & 0 \\
0 & \beta & 0 \\
0 & 0 & -(1+\beta)
\end{array}\right), \quad \sigma^{0}(\lambda)=\frac{2 E}{3}(2+\beta) \lambda(t)\left(\begin{array}{ccc}
1 & 0 & 0 \\
0 & \alpha & 0 \\
0 & 0 & 0
\end{array}\right),
$$

where $\lambda=\varepsilon_{1}$ is a loading control parameter. In fact, Eq. (15) represents a oneparameter family of fundamental paths, and for each value of parameter $\alpha$ or $\beta$ corresponding to a particular loading path, we can investigate the bifurcation or MFC.

\subsection{Bifurcation analysis}

The bifurcation Eq. (5), taking into account the incompressibility condition, $\varepsilon_{1}+\varepsilon_{2}+\varepsilon_{3}=0$, reduces to the following two equations $\Delta \dot{\sigma}_{1}-\sigma_{1} \Delta \dot{\varepsilon}_{1}=0$ and $\Delta \dot{\sigma}_{2}-\sigma_{2} \Delta \dot{\varepsilon}_{2}=0$. The latter, combined with the elastic constitutive Eq. (13), written in their rate form, lead to the following two-equation system:

$$
K\left[\begin{array}{c}
\Delta \dot{\varepsilon}_{1} \\
\Delta \dot{\varepsilon}_{2}
\end{array}\right]=0, \quad \text { with } K=\left[\begin{array}{cc}
\frac{4 E}{3}-\sigma_{1} & \frac{2 E}{3} \\
\frac{2 E}{3} & \frac{4 E}{3}-\sigma_{2}
\end{array}\right]
$$

The above linear algebraic system results in an eigenvalue problem, in which the bifurcation condition necessarily involves the singularity of matrix $K$, leading to

$$
\operatorname{det} K=\alpha X^{2}-\frac{4 E}{3}(1+\alpha) X+3\left(\frac{2 E}{3}\right)^{2}=0
$$


with $X=\sigma_{1}$ and $\sigma_{2}=\alpha \sigma_{1}$. The above second-order polynomial equation has two real roots that are closed-form expressions of the elastic properties (i.e., here the Young modulus) and the loading path parameter ( $\alpha$ or $\beta$ ). As usual in bifurcation analysis, we restrict attention to the lowest critical load, which corresponds to the following critical state in terms of stress and strain:

$$
\left\{\begin{array}{l}
\sigma_{1}^{c}=\frac{2 E}{3 \alpha}\left[(1+\alpha)-\sqrt{\alpha^{2}-\alpha+1}\right] \\
\sigma_{2}^{c}=\frac{2 E}{3}\left[(1+\alpha)-\sqrt{\alpha^{2}-\alpha+1}\right]
\end{array}, \quad\left\{\begin{array}{l}
\varepsilon_{1}^{c}=\frac{(2-\alpha)}{3 \alpha}\left[(1+\alpha)-\sqrt{\alpha^{2}-\alpha+1}\right] \\
\varepsilon_{2}^{c}=\frac{(2 \alpha-1)}{3 \alpha}\left[(1+\alpha)-\sqrt{\alpha^{2}-\alpha+1}\right] .
\end{array}\right.\right.
$$

With these expressions, we can obtain the critical state for the whole range of loading paths of interest; Table 1 reports the critical stresses and strains for three typical loading paths corresponding to uniaxial tension (UT) $(\alpha=0$ or $\beta=-1 / 2)$, plane-strain tension (PT) $(\alpha=1 / 2$ or $\beta=0)$, and $\operatorname{EBE}(\alpha=1$ or $\beta=1)$.

\subsection{Maximum force conditions}

For the reasons discussed previously, focus is confined to the maximum force condition along the major strain direction. Therefore, Eq. (6) along with the incompressibility condition leads to

$$
\dot{\sigma}_{1}-\sigma_{1} \dot{\varepsilon}_{1}=0
$$

Using the constitutive Eq. (13) and the condition of proportional loading $\dot{\varepsilon}_{2}=\beta \dot{\varepsilon}_{1}$, we obtain the critical state in terms of stress and strain:

$$
\left\{\begin{array}{l}
\sigma_{1}^{c}=\frac{2 E}{3}(2+\beta) \\
\sigma_{2}^{c}=\frac{2 E}{3}(1+2 \beta)
\end{array}, \quad\left\{\begin{array}{l}
\varepsilon_{1}^{c}=1 \\
\varepsilon_{2}^{c}=\beta
\end{array}\right.\right.
$$

Again, the whole range of loading paths can be investigated with the expressions above; for comparison purposes, we specify in Table 2 the corresponding critical loads for the same particular loading paths as for the bifurcation analysis.

Table 1. Critical elastic bifurcation states for three typical loading paths.

\begin{tabular}{cccc}
\hline & \multicolumn{3}{c}{ Loading path } \\
\cline { 2 - 4 } Critical state & UT & PT & EBE \\
\cline { 2 - 4 } & $\beta=-1 / 2$ & $\beta=0$ & $\beta=1$ \\
\hline$\sigma_{1}^{c}$ & $E$ & $2 E(1-1 / \sqrt{3})$ & $2 E / 3$ \\
$\sigma_{2}^{c}$ & 0 & $E(1-1 / \sqrt{3})$ & $2 E / 3$ \\
$\varepsilon_{1}^{c}$ & 1 & $\sqrt{3}(\sqrt{3}-1) / 2$ & $1 / 3$ \\
$\varepsilon_{2}^{c}$ & $-1 / 2$ & 0 & $1 / 3$ \\
\hline
\end{tabular}


Table 2. Critical elastic states associated with the MFC for three typical loading paths.

\begin{tabular}{cccc}
\hline & \multicolumn{3}{c}{ Loading path } \\
\cline { 2 - 4 } Critical state & $\mathrm{UT}$ & $\mathrm{PT}$ & $\mathrm{EBE}$ \\
\cline { 2 - 4 }$\beta=-1 / 2$ & $\beta=0$ & $\beta=1$ \\
\hline$\sigma_{1}^{c}$ & $E$ & $4 E / 3$ & $2 E$ \\
$\sigma_{2}^{c}$ & 0 & $2 E / 3$ & $2 E$ \\
$\varepsilon_{1}^{c}$ & 1 & 1 & 1 \\
$\varepsilon_{2}^{c}$ & $-1 / 2$ & 0 & 1 \\
\hline
\end{tabular}

\subsection{Discussion}

As shown previously, the condition of simultaneous maximum of the forces is only possible for two typical loading paths (i.e., UT and EBE). For these two loading paths, this condition also amounts to that of the maximum force along the major strain direction, as reported in Table 2.

The results yielded by the bifurcation analysis and MFC can be compared through their respective equations (i.e., Eqs. (18) and (20)) as well as through Tables 1 and 2. For a wide range of loading paths corresponding to $\beta \in[-1 / 2,1]$, Fig. 2 compares the bifurcation and MFC results in terms of both critical strains and the associated critical stresses. Although less commonly used, the stress representation of critical states is also shown here for subsequent comparison with the elasto-plastic case.

One can observe that except for the case of UT, the results given by the bifurcation analysis (solid lines) differ significantly from those given by the MFC (dashed lines). The critical loads predicted by bifurcation are typically lower than those of the MFC. Thus, as expected, this simple illustrative problem confirms that these
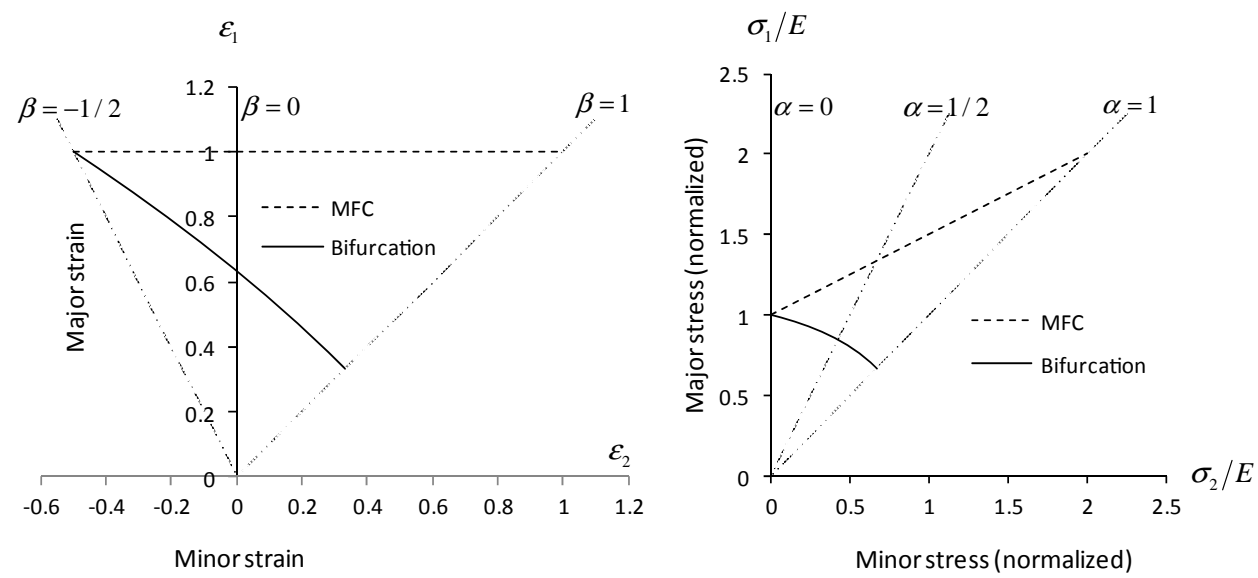

Fig. 2. Comparison of elastic critical strains/stresses given by bifurcation and MFC. 
two approaches represent intrinsically distinct concepts. Indeed, while bifurcation is associated with loss of uniqueness, it seems more difficult to provide theoretical foundations (e.g., stability, loss of uniqueness) for the MFC concept.

Note that the validity of these observations is not limited to the case of incompressibility; the same analyses without volume conservation restrictions have been carried out and show similar differences between the two approaches.

Note also that even though the bifurcation analysis results coincide with those of MFC for UT, this does not imply that for this particular loading path the two concepts are equivalent. In elasticity and conventional elasto-plasticity, bifurcation and MFC yield the same critical loads for UT, but this is not necessarily the case for other constitutive models (e.g., elasto-viscoplasticity).

\section{Investigation in Elasto-Plasticity}

In this section, the two approaches (i.e., bifurcation and MFC) previously investigated in elasticity will be further explored for a more realistic material model, commonly used in sheet metal forming applications. The same assumptions as before are made along with the same notations and definitions.

\subsection{Elasto-plastic fundamental equilibrium path}

We consider in what follows a general form of elasto-plastic constitutive equations with incompressible elasticity and isotropic hardening. This constitutive framework, despite its simplicity, can include a large variety of work hardening models as typically used in sheet metal forming. Using the additive decomposition of the strain rate tensor into its elastic and plastic parts $\dot{\varepsilon}^{e}$ and $\dot{\varepsilon}^{p}$, respectively, the elasticity law reads

$$
\dot{\varepsilon}^{e}=\dot{\varepsilon}-\dot{\varepsilon}^{p}=\frac{3}{2 E} \dot{\boldsymbol{\sigma}}-\frac{1}{2 E} \operatorname{tr}(\dot{\boldsymbol{\sigma}}) \mathbf{I}_{2}
$$

The plastic strain rate tensor is given by the usual associative flow rule:

$$
\dot{\varepsilon}^{p}=\dot{p} \frac{\partial F}{\partial \boldsymbol{\sigma}},
$$

where $\dot{p}$ denotes the plastic multiplier and $F$ the yield surface, here given by

$$
F=\sigma_{\mathrm{eq}}-Y\left(\bar{\varepsilon}^{p}\right)
$$

in which $\sigma_{\mathrm{eq}}=\sqrt{3 / 2 \sigma^{\prime}: \sigma^{\prime}}$ is the von Mises equivalent stress, function of the deviatoric stress tensor $\boldsymbol{\sigma}^{\prime} . Y$ is the yield stress describing isotropic hardening, which is a function of the equivalent plastic strain $\bar{\varepsilon} p$, with $\dot{\bar{\varepsilon}}^{p}=\sqrt{2 / 3 \dot{\varepsilon}^{p}: \dot{\varepsilon}^{p}}$.

Making use of the consistency condition, the plastic multiplier can be determined along with the elasto-plastic tangent modulus in the following particular tensorial form:

$$
\frac{3}{2 E} \dot{\boldsymbol{\sigma}}-\frac{1}{2 E} \operatorname{tr}(\dot{\boldsymbol{\sigma}}) \mathbf{I}_{2}=\dot{\varepsilon}-\frac{3}{2} \frac{E}{(E+h)} \frac{1}{\sigma_{\mathrm{eq}}^{2}}\left(\boldsymbol{\sigma}^{\prime} \otimes \boldsymbol{\sigma}^{\prime}\right): \dot{\varepsilon}
$$


in which $h=d Y / d \bar{\varepsilon}^{p}$ is the scalar hardening modulus. In terms of components we have

$$
\left\{\begin{array}{l}
\dot{\sigma}_{1}=\frac{2 E}{3}\left(2 \dot{\varepsilon}_{1}+\dot{\varepsilon}_{2}\right)-\frac{E}{(E+h)} \frac{E}{\sigma_{\mathrm{eq}}^{2}} \sigma_{1}\left(\sigma_{1} \dot{\varepsilon}_{1}+\sigma_{2} \dot{\varepsilon}_{2}\right) \\
\dot{\sigma}_{2}=\frac{2 E}{3}\left(2 \dot{\varepsilon}_{2}+\dot{\varepsilon}_{1}\right)-\frac{E}{(E+h)} \frac{E}{\sigma_{\mathrm{eq}}^{2}} \sigma_{2}\left(\sigma_{1} \dot{\varepsilon}_{1}+\sigma_{2} \dot{\varepsilon}_{2}\right) .
\end{array}\right.
$$

The material response consists of two stages; an elastic stage followed by an elastoplastic regime. Note that the elastic case investigated in the previous section can be recovered in the limit of $h \rightarrow+\infty$ in Eqs. (24) or (25), whereby the uniaxial tangent modulus $E_{T}=E h /(E+h)$ consistently tends toward the Young modulus $E$. In the same way as before, it can be shown that a proportional loading in terms of strain components with parameter $\beta$ results in proportional stress components with ratio $\alpha$, with the same relation (14) between $\alpha$ and $\beta$. Making use of these proportionality factors, the constitutive Eq. (25) can be rewritten in a more compact form:

$$
\left\{\begin{array}{l}
\dot{\sigma}_{1}=\frac{2 E_{T}}{3}(2+\beta) \dot{\varepsilon}_{1} \\
\dot{\sigma}_{2}=\frac{2 E_{T}}{3}(1+2 \beta) \dot{\varepsilon}_{1} .
\end{array}\right.
$$

For a biaxial loading controlled by an increasing parameter $\lambda(t)$, the fundamental solution in terms of strain $\varepsilon^{0}(\lambda)$ given by Eq. (15) still holds. In terms of stress, however, the expression given by Eq. (15) only holds for the elastic stage of the loading (i.e., for $\lambda(t) \in\left[0, \lambda^{Y}\right]$, with $\lambda^{Y}=\sqrt{3} \sigma_{y} /\left[2 E \sqrt{\beta^{2}+\beta+1}\right]$, and $\sigma_{y}$ the initial yield stress). For the plastic range (i.e., $\lambda(t)>\lambda^{Y}$ ), the fundamental response in terms of stress is given by

$$
\left\{\begin{array}{l}
\sigma_{1}^{0}=\sigma_{1}^{Y}+\frac{2}{3}(2+\beta) \int_{\lambda^{Y}}^{\lambda(t)} E_{T} d \lambda \\
\sigma_{2}^{0}=\sigma_{2}^{Y}+\frac{2}{3}(1+2 \beta) \int_{\lambda^{Y}}^{\lambda(t)} E_{T} d \lambda
\end{array}\right.
$$

where $\sigma_{1}^{Y}=\sigma_{y} / \sqrt{\alpha^{2}-\alpha+1}$ and $\sigma_{2}^{Y}=\alpha \sigma_{1}^{Y}$.

For a linear hardening model, for which $h$ and hence $E_{T}$ are constant, a closedform solution can be obtained in straightforward manner. In the general case of nonlinear hardening models, the following convenient relationship can be derived:

$$
E \bar{\varepsilon}^{p}+Y\left(\bar{\varepsilon}^{p}\right)=\frac{2 E \sqrt{\beta^{2}+\beta+1}}{\sqrt{3}} \varepsilon_{1} .
$$




\subsection{Elasto-plastic bifurcation analysis}

We consider again the rate form of the equilibrium equations and the associated bifurcation problem discussed in Sec. 2. Within the framework of elasto-plasticity, the governing bifurcation equations are:

$$
K\left[\begin{array}{c}
\Delta \dot{\varepsilon}_{1} \\
\Delta \dot{\varepsilon}_{2}
\end{array}\right]=0 \quad \text { with } K=\left[\begin{array}{cc}
\frac{4 E}{3}-\sigma_{1}-\frac{E^{2}}{(E+h)} \frac{\sigma_{1}^{2}}{\sigma_{\mathrm{eq}}^{2}} & \frac{2 E}{3}-\frac{E^{2}}{(E+h)} \frac{\sigma_{1} \sigma_{2}}{\sigma_{\mathrm{eq}}^{2}} \\
\frac{2 E}{3}-\frac{E^{2}}{(E+h)} \frac{\sigma_{1} \sigma_{2}}{\sigma_{\mathrm{eq}}^{2}} & \frac{4 E}{3}-\sigma_{2}-\frac{E^{2}}{(E+h)} \frac{\sigma_{2}^{2}}{\sigma_{\mathrm{eq}}^{2}}
\end{array}\right]
$$

and the condition of vanishing determinant of matrix $K$ leads to the following characteristic equation:

$$
\operatorname{det} K=\alpha X^{2}-(1+\alpha)(a-b \alpha) X+a E_{T}=0
$$

in which $X=\sigma_{1}, \sigma_{2}=\alpha \sigma_{1}, a=4 E / 3$ and $b=E^{2} /\left[(E+h)\left(\alpha^{2}-\alpha+1\right)\right]$. Among the two real roots of the second-order polynomial above, the one corresponding to the lowest critical load leads to

$$
\left\{\begin{array}{l}
\sigma_{1}^{c}=\frac{(1+\alpha)(a-b \alpha)}{2 \alpha}\left(1-\sqrt{1-\frac{4 \alpha a E_{T}}{(1+\alpha)^{2}(a-b \alpha)^{2}}}\right) . \\
\sigma_{2}^{c}=\alpha \sigma_{1}^{c}
\end{array}\right.
$$

Once again, when $h \rightarrow+\infty$, we have $b \rightarrow 0$, and the elastic bifurcation problem with its associated critical states is exactly recovered. For finite values of the hardening modulus, the elasto-plastic critical stresses are given by Eq. (31) as functions of the critical hardening modulus, denoted $h^{c}$. The determination of this critical hardening modulus, which completely defines the critical state, generally requires a nonlinear equation to be solved. The latter is given by the yield condition $F=0$, which can be rewritten as

$$
\sigma_{1}^{c} \sqrt{\alpha^{2}-\alpha+1}=Y\left(\bar{\varepsilon}_{c}^{p}\right) .
$$

In the equation above, in which $\sigma_{1}^{c}$ is replaced by its expression from Eq. (31), the main unknown to be determined is $\bar{\varepsilon}_{c}^{p}$. This allows us to define $h^{c}=Y^{\prime}\left(\bar{\varepsilon}_{c}^{p}\right)$ and then the corresponding critical stresses from Eq. (31). Making use of Eqs. (28) and (32), the critical strains can subsequently be obtained from the following equations:

$$
\left\{\begin{array}{l}
\varepsilon_{1}^{c}=\frac{\sqrt{3}}{2 E \sqrt{\beta^{2}+\beta+1}}\left[E \bar{\varepsilon}_{c}^{p}+\sigma_{1}^{c} \sqrt{\alpha^{2}-\alpha+1}\right] . \\
\varepsilon_{2}^{c}=\beta \varepsilon_{1}^{c}
\end{array}\right.
$$

Similarly to the elastic case investigated in Sec. 3.2, Eqs. (31)-(33) allow us to determine the critical bifurcation states for the whole range of loading paths and 
Table 3. Critical elasto-plastic bifurcation states for three typical loading paths.

\begin{tabular}{cccc}
\hline & \multicolumn{4}{c}{ Loading path } \\
\cline { 2 - 4 } Critical state & $\mathrm{UT}$ & $\mathrm{PT}$ & $\mathrm{EBE}$ \\
\cline { 2 - 4 } & $\beta=-1 / 2$ & $\beta=0$ & $\beta=1$ \\
\hline$\sigma_{1}^{c}$ & $E_{T}^{c}$ & $E+E_{T}^{c}-\sqrt{E^{2}-(2 / 3) E E_{T}^{c}+E_{T}^{c 2}}$ & $1 / 3\left(E+3 E_{T}^{c}\right)-1 / 3\left|E-3 E_{T}^{c}\right|$ \\
$\sigma_{2}^{c}$ & 0 & $\sigma_{1}^{c} / 2$ & $\sigma_{1}^{c}$ \\
$\varepsilon_{1}^{c}$ & $\bar{\varepsilon}_{c}^{p}+E_{T}^{c} / E$ & $\sqrt{3} / 2\left(\bar{\varepsilon}_{c}^{p}+\sqrt{3} \sigma_{1}^{c} / 2 E\right)$ & $1 / 2\left(\bar{\varepsilon}_{c}^{p}+\sigma_{1}^{c} / E\right)$ \\
$\varepsilon_{2}^{c}$ & $-\varepsilon_{1}^{c} / 2$ & 0 & $\varepsilon_{1}^{c}$ \\
\hline
\end{tabular}

for a variety of nonlinear hardening laws. To illustrate this, Table 3 reports the critical stress and strain states for three typical loading paths.

In Table 3, the results depend on $E_{T}^{c}=E h^{c} / E h^{c}\left(E+h^{c}\right)$ and thus on the critical hardening modulus $h^{c}$, which itself depends on $\bar{\varepsilon}_{c}^{p}$. This critical equivalent plastic strain is determined by solving Eq. (32), which represents a linear or nonlinear algebraic equation depending on the isotropic hardening law selected. For illustration, the resulting critical equivalent plastic strain and hardening modulus, when $h / E \ll 1$, are given in Appendix A for a set of common isotropic hardening models.

Based on the results in Table 3, two observations are interesting to make. The first is that when $h / E \ll 1$, the critical stress for the PT loading path is equivalent to the value of $\sigma_{1}^{c}=4 E_{T}^{c} / 3$. The second is that in $\mathrm{EBE}$, there is a threshold value for the hardening (i.e., $h^{L}=E / 2$ ) under which the critical stress is $\sigma_{1}^{c}=2 E_{T}^{c}$, and beyond which it is equal to $\sigma_{1}^{c}=2 E / 3$, regardless of plasticity. For hardening moduli beyond this threshold, the critical stresses for EBE found in elasticity (see Sec. 3, Table 1) are recovered; however, the associated critical strains are still affected by plasticity.

\section{3. $M F C$}

The discussions in Secs. 2 and 3 regarding the range of applicability of the MFC still hold in the context of elasto-plasticity. The maximum force condition along the major strain direction, given by Eq. (19), together with the constitutive Eq. (26) leads to

$$
\left\{\begin{array}{l}
\sigma_{1}^{c}=\frac{2 E_{T}^{c}}{3}(2+\beta) \\
\sigma_{2}^{c}=\frac{2 E_{T}^{c}}{3}(1+2 \beta) .
\end{array}\right.
$$

Note that the resulting elasto-plastic critical stresses are given by expressions that are nearly identical to those obtained in the elastic case (see Eq. (20)), whereby Young's modulus $E$ is replaced by the elasto-plastic scalar tangent modulus $E_{T}^{c}$. 
Table 4. Critical elasto-plastic states given by MFC for three typical loading paths.

\begin{tabular}{cccc}
\hline & \multicolumn{3}{c}{ Loading path } \\
\cline { 2 - 4 } Critical state & $\mathrm{UT}$ & $\mathrm{PT}$ & $\mathrm{EBE}$ \\
\cline { 2 - 4 } & $\beta=-1 / 2$ & $\beta=0$ & $\beta=1$ \\
\hline$\sigma_{1}^{c}$ & $E_{T}^{c}$ & $4 E_{T}^{c} 3$ & $2 E_{T}^{c}$ \\
$\sigma_{2}^{c}$ & 0 & $2 E_{T}^{c} / 3$ & $2 E_{T}^{c}$ \\
$\varepsilon_{1}^{c}$ & $\bar{\varepsilon}_{c}^{p}+E_{T}^{c} / E$ & $\sqrt{3} \bar{\varepsilon}_{c}^{p} / 2+E_{T}^{c} / E$ & $\bar{\varepsilon}_{c}^{p} / 2+E_{T}^{c} / E$ \\
$\varepsilon_{2}^{c}$ & $-\varepsilon_{1}^{c} / 2$ & 0 & $\varepsilon_{1}^{c}$ \\
\hline
\end{tabular}

The critical strain evaluation also relies on the solution of Eqs. (32) and (33), in which the critical hardening modulus $h^{c}$ needs to be determined first.

For comparison purposes, Table 4 reports the critical stresses and strains obtained with the MFC for three particular loading paths.

Again, the quantitative evaluation of the expressions in Table 4 depends on the hardening law selected. For the case when $h / E \ll 1$, we provide in Appendix A, the associated critical equivalent plastic strain and hardening modulus for a set of popular isotropic hardening models.

\subsection{Discussion}

Before comparing the results of bifurcation and MFC within the context of elastoplasticity, it is first verified that in the limit of $h \rightarrow+\infty$, all results consistently recover those obtained in Sec. 3 for the elastic case.

Looking at the critical loads due to bifurcation, Eq. (31), and those predicted by the MFC, Eq. (34), reveals an overall difference depending on the hardening model and loading path considered. In the particular UT loading path, the results of bifurcation and MFC coincide, regardless of the hardening law. For the PT loading path, the results of the two approaches differ for general hardening models, but the bifurcation critical state tends to the MFC when $h / E \ll 1$. For the EBE loading path, the results of the bifurcation analysis and MFC differ for $h>h^{L}=E / 2$, while they coincide below this hardening limit (which is the relevant engineering regime, see also Fig. 3 further on).

A common characteristic is that the bifurcation and MFC critical loads for PT and EBE become equivalent under the condition $h / E \ll 1$. Moreover, for the three loading paths UT, PT and EBE, the two approaches provide the same results in the limit of $h / E \rightarrow 0$.

For other loading paths, even in this limit of a small $h / E$ ratio, the results of the two approaches are not necessarily equivalent. To demonstrate this, a plot of the critical loads obtained by the two approaches is given in Fig. 3 for three values of the ratio $h / E$ (i.e., $10^{-1}, 10^{-2}$ and $10^{-3}$ ). Note that the choice of stress space in Fig. 3 proves to be the simplest way to compare the results of the two approaches. Indeed, while the plots in Fig. 3 only depend on the ratio $E_{T} / E$ (or equivalently 


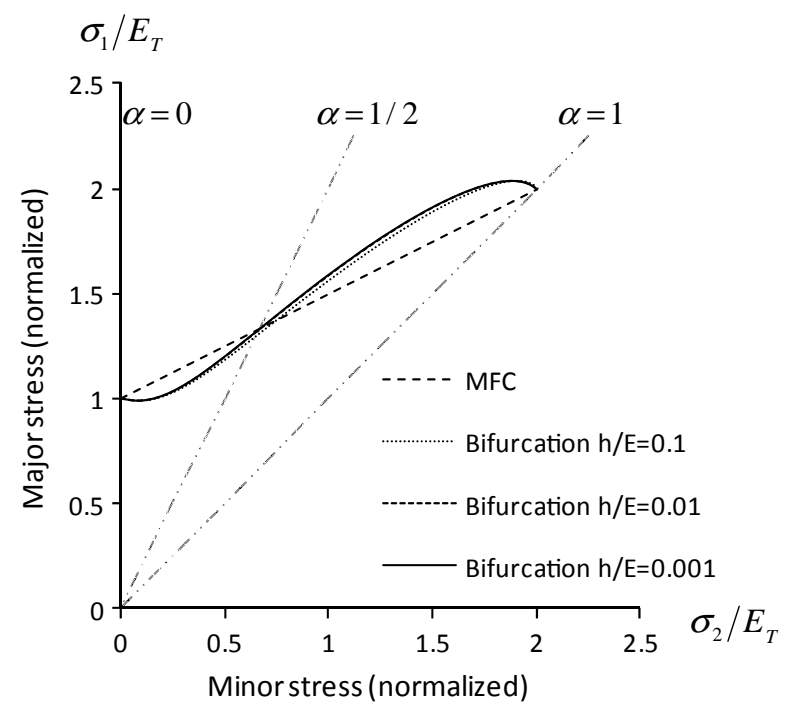

Fig. 3. Comparison between the bifurcation and MFC results for three values of $h / E$.

on the $h / E$ ratio), the alternative representation in terms of critical strains would require the determination of the critical equivalent plastic strain (Eq. (32)), which depends on the material parameters of the selected hardening model.

Another way to quantitatively compare the two approaches when $h / E \ll 1$ is to perform a Taylor series expansion of expression (31), truncated after the first-order terms in $h / E$. This gives the following equivalent formula for the bifurcation critical load:

$$
\sigma_{1}^{c}=\frac{E_{T}^{c}}{(1+\alpha)\left(1-3 \alpha /\left[4\left(\alpha^{2}-\alpha+1\right)\right]\right)} .
$$

This simple formula turns out to be an accurate and useful approximation of the critical stresses, since it is valid for any hardening model as long as $h / E \ll 1$. Comparing this expression with its counterpart given by the MFC, Eq. (34), reveals that they are equal for $\alpha=0, \alpha=1 / 2$ and $\alpha=1$. This means that the results of the two approaches, in the limit of $h / E \ll 1$, only coincide for the UT, PT and EBE loading paths.

\section{Special Case of Rigid-Plasticity}

The special case of rigid-plasticity is of particular importance because of its wide use in sheet metal forming applications. Most formability criteria based on the maximum force principle were therefore developed in this context. Sheet metal forming involves large plastic deformations, which justifies neglecting the elastic strains. Moreover, the convenient framework of rigid-plasticity enables closed-form 
expressions for the MFC. Developing this special case will allow us to compare the obtained results with some of the criteria available in the literature.

\subsection{Fundamental equilibrium path in rigid-plasticity}

The basic equations for rigid-plasticity with isotropic hardening can be recovered from Sec. 4.1 in the limit of $E \rightarrow+\infty$ (which also implies that $E_{T} \rightarrow h$ ). The plastic strain rate, equal here to the total strain rate, is given by the same flow rule (22), with the plastic multiplier given by $\dot{p}=\dot{\bar{\varepsilon}}^{p}=\dot{\bar{\varepsilon}}$. Therefore, the rigid-plasticity constitutive equations are simply deduced from Eq. (24), as a special case, under the tensorial form

$$
\dot{\varepsilon}=\frac{3}{2} \frac{1}{\sigma_{\text {eq }}^{2}}\left(\boldsymbol{\sigma}^{\prime} \otimes \boldsymbol{\sigma}^{\prime}\right): \dot{\varepsilon},
$$

or in terms of stress and strain components:

$$
\left\{\begin{array}{l}
\left(2 \dot{\varepsilon}_{1}+\dot{\varepsilon}_{2}\right)=\frac{3}{2 \sigma_{\mathrm{eq}}^{2}} \sigma_{1}\left(\sigma_{1} \dot{\varepsilon}_{1}+\sigma_{2} \dot{\varepsilon}_{2}\right) \\
\left(2 \dot{\varepsilon}_{2}+\dot{\varepsilon}_{1}\right)=\frac{3}{2 \sigma_{\mathrm{eq}}^{2}} \sigma_{2}\left(\sigma_{1} \dot{\varepsilon}_{1}+\sigma_{2} \dot{\varepsilon}_{2}\right) .
\end{array}\right.
$$

Again, the proportional loading conditions previously applied result in the same relation (14) between parameters $\alpha$ and $\beta$. With these proportionality factors, the following relations are directly obtained from Eq. (26), in the limit of $E \rightarrow+\infty$ :

$$
\left\{\begin{array}{l}
\dot{\sigma}_{1}=\frac{2 h}{3}(2+\beta) \dot{\varepsilon}_{1} \\
\dot{\sigma}_{2}=\frac{2 h}{3}(1+2 \beta) \dot{\varepsilon}_{1} .
\end{array}\right.
$$

Integrating these equations provides the fundamental solution in the same way as given by Eq. (27), with no preliminary elastic stage (i.e., $\lambda^{Y}=0$ ), and with $E_{T}$ replaced by the hardening modulus $h$. An analytical solution exists for a linear hardening model, while nonlinear models require numerical integration. In all cases, the following convenient relationship can be derived:

$$
\bar{\varepsilon}=\frac{2 \sqrt{\beta^{2}+\beta+1}}{\sqrt{3}} \varepsilon_{1} .
$$

\subsection{Bifurcation analysis}

The bifurcation analysis in the context of rigid-plasticity is slightly different from the one carried out previously. Indeed, the constitutive Eq. (36) now reveal a singular tangent modulus. As a result, Eq. (37) is a linear system in which the two equations are not independent. Therefore another equation is required to complete 
the bifurcation problem. The first equation for the bifurcation analysis is obtained by combining the two equations in (37), which gives:

$$
\left(\sigma_{1}-2 \sigma_{2}\right) \Delta \dot{\varepsilon}_{1}+\left(2 \sigma_{1}-\sigma_{2}\right) \Delta \dot{\varepsilon}_{2}=0
$$

The second bifurcation equation is derived starting from the consistency condition $\dot{F}=\dot{\sigma}_{\text {eq }}-\dot{Y}(\bar{\varepsilon})=0$. By replacing the equivalent strain rate by the expression for the plastic multiplier $\dot{p}=\sigma: \dot{\varepsilon} / \sigma_{\text {eq }}$, and making use of the equilibrium Eq. (9), the consistency condition leads to

$$
\sigma_{1}\left(2 \sigma_{1}-\sigma_{2}-2 h\right) \Delta \dot{\varepsilon}_{1}+\sigma_{2}\left(2 \sigma_{2}-\sigma_{1}-2 h\right) \Delta \dot{\varepsilon}_{2}=0 .
$$

The matrix associated with this bifurcation problem (Eqs. (40) and (41)) is

$$
K=\left[\begin{array}{cc}
\sigma_{1}-2 \sigma_{2} & 2 \sigma_{1}-\sigma_{2} \\
\sigma_{1}\left(2 \sigma_{1}-\sigma_{2}-2 h\right) & \sigma_{2}\left(2 \sigma_{2}-\sigma_{1}-2 h\right)
\end{array}\right]
$$

and the vanishing of its determinant provides the critical state:

$$
\left\{\begin{array}{l}
\sigma_{1}^{c}=\frac{4 h^{c}\left(\alpha^{2}-\alpha+1\right)}{(1+\alpha)\left(4 \alpha^{2}-7 \alpha+4\right)} . \\
\sigma_{2}^{c}=\alpha \sigma_{1}^{c}
\end{array}\right.
$$

The determination of the critical hardening modulus in Eq. (43) is achieved by solving the following equation given by the yield function $F=0$ :

$$
\sigma_{1}^{c} \sqrt{\alpha^{2}-\alpha+1}=Y\left(\bar{\varepsilon}^{c}\right)
$$

in which the critical stress $\sigma_{1}^{c}$ is replaced by its expression in terms of $h^{c}=d Y /\left.d \bar{\varepsilon}\right|_{\bar{\varepsilon}^{c}}$. Finally, the critical strains are given by

$$
\left\{\begin{array}{l}
\varepsilon_{1}^{c}=\frac{\sqrt{3} \bar{\varepsilon}^{c}}{2 \sqrt{\beta^{2}+\beta+1}} . \\
\varepsilon_{2}^{c}=\beta \varepsilon_{1}^{c}
\end{array}\right.
$$

It is remarkable that Eqs. (43) and (44) are exactly the expressions given by the Swift'52 diffuse necking criterion in rigid-plasticity, which can be rewritten as

$$
\left.\frac{1}{Y} \frac{d Y}{d \bar{\varepsilon}}\right|_{\bar{\varepsilon}^{c}}=\frac{(1+\alpha)\left(4 \alpha^{2}-7 \alpha+4\right)}{4\left(\alpha^{2}-\alpha+1\right)^{3 / 2}} .
$$

This criterion has been frequently used in the literature to construct FLDs at diffuse necking for metal sheets. Table 5 gives the critical stress and strain states for the three particular loading paths previously investigated.

\subsection{Maximum force conditions}

The preceding discussions concerning the relevance and limitations of some MFC are still valid in the framework of rigid-plasticity. Therefore, the maximum force 
Table 5. Critical rigid-plastic bifurcation states for three typical loading paths.

\begin{tabular}{cccc}
\hline & \multicolumn{3}{c}{ Loading path } \\
\cline { 2 - 4 } Critical state & $h^{c}$ & $\beta=0$ & PT \\
\cline { 2 - 4 } & $\beta=-1 / 2$ & $4 h^{c} / 3$ & $2 h^{c}$ \\
\hline$\sigma_{1}^{c}$ & 0 & $2 h^{c} / 3$ & $2 h^{c}$ \\
$\sigma_{2}^{c}$ & $\bar{\varepsilon}^{c}$ & $\sqrt{3} \bar{\varepsilon}^{c} / 2$ & $\bar{\varepsilon}^{c} / 2$ \\
$\varepsilon_{1}^{c}$ & $-\bar{\varepsilon}^{c} / 2$ & 0 & $\bar{\varepsilon}^{c} / 2$ \\
$\varepsilon_{2}^{c}$ & & & \\
\hline
\end{tabular}

Table 6. Critical rigid-plastic states given by the MFC for three typical loading paths.

\begin{tabular}{cccc}
\hline & \multicolumn{3}{c}{ Loading path } \\
\cline { 2 - 4 } Critical state & $h^{c}$ & $\mathrm{UT}$ & $\mathrm{EBE}$ \\
\cline { 2 - 4 } & $\beta=-1 / 2$ & $\beta=0$ & $\beta=1$ \\
\hline$\sigma_{1}^{c}$ & 0 & $4 h^{c} / 3$ & $2 h^{c}$ \\
$\sigma_{2}^{c}$ & $\bar{\varepsilon}^{c}$ & $2 h^{c} / 3$ & $2 h^{c}$ \\
$\varepsilon_{1}^{c}$ & $-\sqrt{3} \bar{\varepsilon}^{c} / 2$ & $\bar{\varepsilon}^{c} / 2$ \\
$\varepsilon_{2}^{c}$ & $-\bar{\varepsilon}^{c} / 2$ & 0 & $\bar{\varepsilon}^{c} / 2$ \\
\hline
\end{tabular}

condition along the major strain direction, given by Eq. (19), combined with the constitutive Eq. (38) gives

$$
\left\{\begin{array}{l}
\sigma_{1}^{c}=\frac{2 h^{c}}{3}(2+\beta) \\
\sigma_{2}^{c}=\frac{2 h^{c}}{3}(1+2 \beta) .
\end{array}\right.
$$

These rigid-plastic critical stresses are given by expressions similar to those of Eq. (34), in which $E_{T}^{c}$ is replaced by $h^{c}$. The corresponding critical strains can be obtained by solving Eqs. (44) and (45), in which the critical hardening modulus $h^{c}$ needs to be first determined. For comparison purposes, Table 6 provides the rigid-plastic critical stresses and strains obtained with the MFC for three particular loading paths.

The results of Table 6 , which are identical to those of Table 5 for these three specific loading paths, depend on the particular hardening law adopted. For completeness, the corresponding critical equivalent strain and hardening modulus for a set of common isotropic hardening models are given in Appendix A.

For more loading paths, Fig. 4 shows FLDs at diffuse necking obtained with the bifurcation approach and the MFC for a set of hardening models. These FLDs apply to copper foils described by rigid-plastic constitutive equations (or elasto-plastic models with $h / E \ll 1)$. The material parameters corresponding to four hardening models (i.e., linear, Hollomon, Swift and Voce laws) have been identified using a 

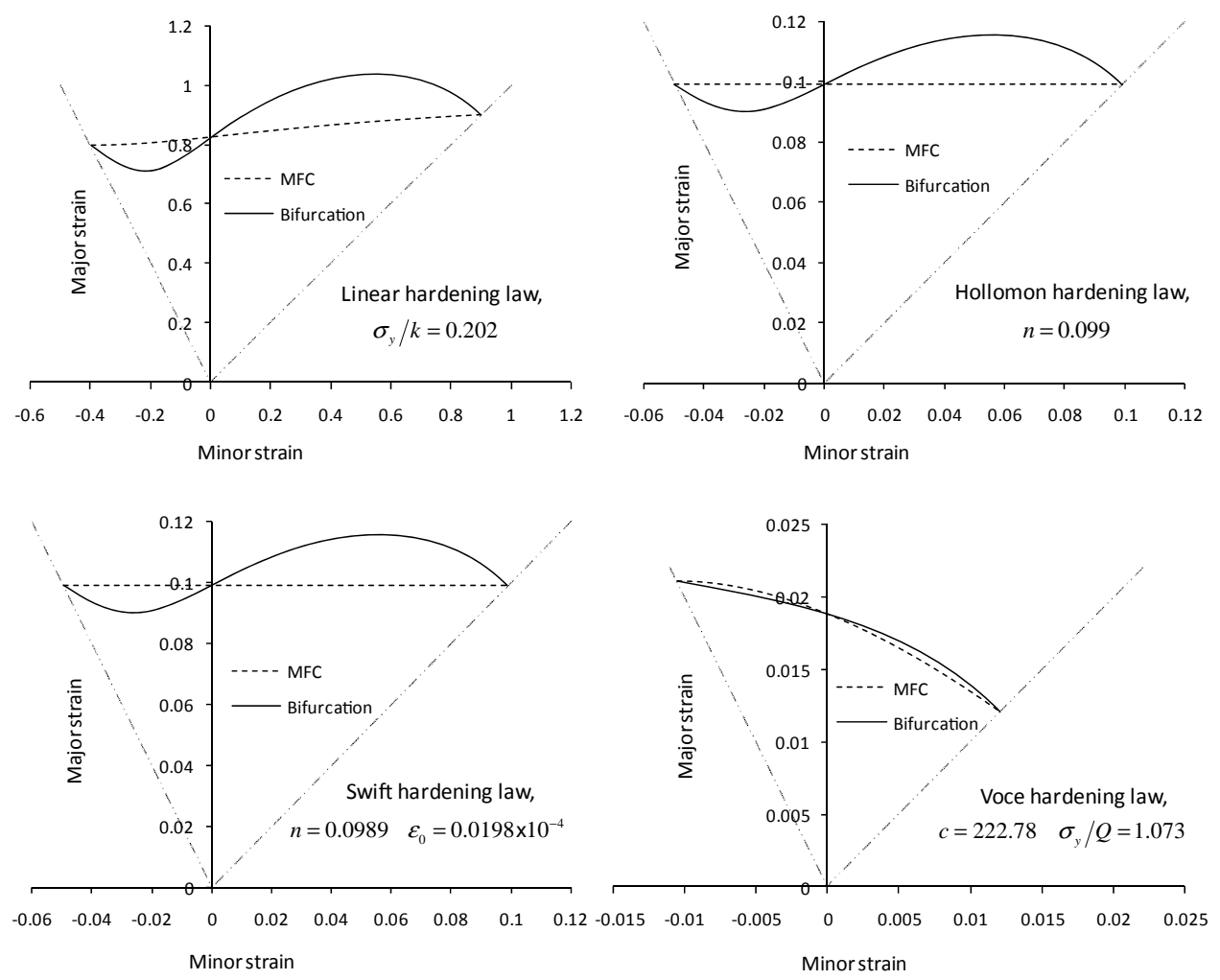

Fig. 4. FLDs at diffuse necking given by bifurcation and MFC for rigid-plastic models (or elastoplastic models with $h / E \ll 1$ ) and four frequently used hardening laws.

UT experiment provided in van der Sluis et al. [2011], and the relevant values of these parameters are reported in Fig. 4. The stress-strain responses for the UT test are depicted in Fig. 5, which shows the relative ability of these hardening models to fit the experimental data.

Figure 4 reveals the impact of the selected material model on necking predictions. Both the shape and the level of the FLDs are strongly affected by the material model. As can be expected, a linear hardening model leads to unrealistically high limit strains, as opposed to the Voce model, which is a rapidly saturating hardening law providing the lowest FLDs. The intermediate hardening models of Hollomon and Swift, which give similar results, correspond to more commonly observed FLDs, and this is also supported by the fact that they fit the experimental UT data in Fig. 5 better.

\subsection{Discussion}

It is obvious that some of the results in this section could be recovered from the analyses carried out on elasto-plasticity in Sec. 4, by simply taking the limit of 


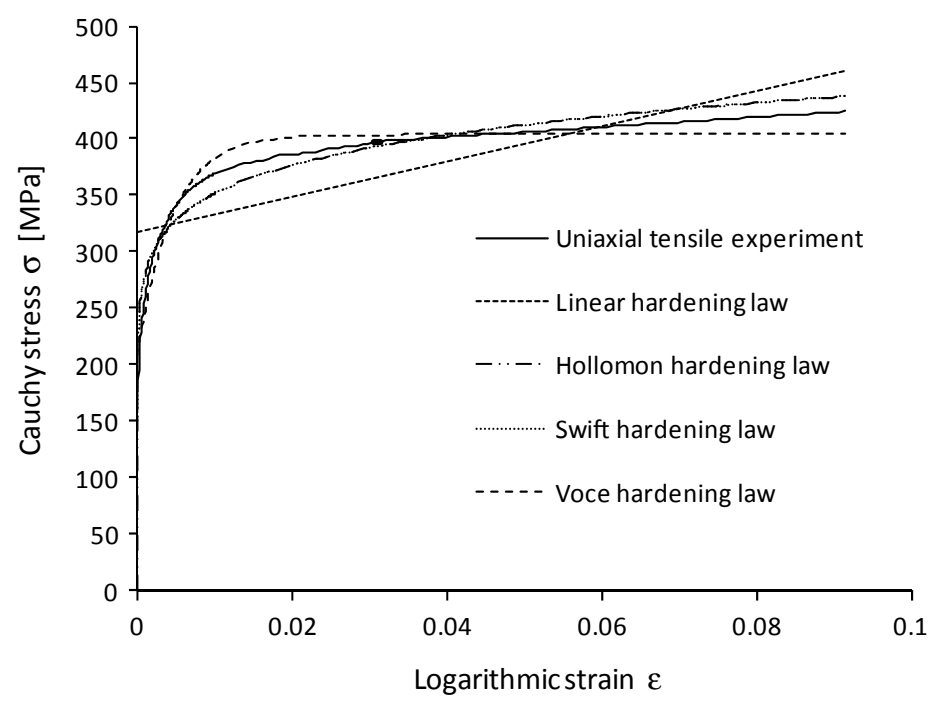

Fig. 5. Stress-strain responses given by four popular isotropic hardening models as identified on a UT test.

$E \rightarrow+\infty$. These include the constitutive equations, the fundamental equilibrium path and the MFC results. However, this is not the case for the bifurcation equations, which had to be specifically derived for rigid-plasticity.

The comparison between the bifurcation analysis (Eq. (43)) and the MFC (Eq. (47)) reveals that, for general hardening laws and loading paths, the associated critical states differ. Equating expression (43) and (47) shows that they only coincide for $\alpha=0, \alpha=1 / 2$ and $\alpha=1$, i.e., for the three particular loading paths reported in Tables 5 and 6 .

It is also worth noting that for elasto-plastic constitutive models coupled with hardening laws such that $h / E \ll 1$, the analysis results in terms of critical states given by the bifurcation analysis or MFC are equivalent to their counterparts obtained within the framework of rigid-plasticity.

Finally, it is important to emphasize the conclusions made on one of the most popular diffuse necking criteria for stretched metal sheets, i.e., the Swift [1952] criterion for rigid-plasticity. This formula has long been thought to originate from the maximum force principle. In the present contribution, we show that this celebrated expression is rather justified within the bifurcation theory. The Swift'52 criterion was originally derived assuming the simultaneous maximum of forces, which is known in the literature not to hold. The fact that we recover the original expression due to Swift on the basis of a bifurcation analysis, not assuming any maximum force, is a remarkable coincidence. This coincidence is only due to the similarity of the set of equations underpinning both analyses. Indeed, these sets of equations take a similar form; the only difference is that the main unknown $\left(\dot{\varepsilon}_{1}, \dot{\varepsilon}_{2}\right)$ is replaced by $\left(\Delta \dot{\varepsilon}_{1}, \Delta \dot{\varepsilon}_{2}\right)$ when bifurcation is dealt with. 


\section{Discussion and Concluding Remarks}

This paper first specified the basic equations underlying the bifurcation analysis and those relating to the maximum force principle, which are independent of the constitutive models used. Some restrictions to the maximum force conditions have been discussed. In particular, the simultaneous occurrence of the maximum of the forces is shown to be limited to some specific loading paths.

Through a preliminary discussion in the framework of elasticity, the difference between the concepts of bifurcation and maximum force principle has been clearly evidenced. Subsequently, these two distinct criteria have been systematically compared for different types of constitutive equations involving a variety of isotropic work-hardening models.

It has been shown that the results obtained in elasto-plasticity (Sec. 4), in terms of fundamental equilibrium path, bifurcation and MFC analyses, all consistently recover those found in elasticity (Sec. 3) in the limit of $h \rightarrow+\infty$. In the same way, they recover, to some extent, the results of rigid-plasticity (Sec. 5) in the limit of $E \rightarrow+\infty$.

An interesting result concerns the elasto-plastic constitutive modeling, in the case $h / E \ll 1$. For material models that fall into this category, the critical states are shown to be equivalent to those obtained in rigid-plasticity. This result is of practical importance, because the rigid-plasticity framework allows for convenient closed-form expressions for the critical states.

One of the main side results of the present contribution is the fact that it was demonstrated that the Swift'52 necking criterion is founded on bifurcation theory rather than any maximum force condition. This provides a better justification for its wide application in the context of sheet metal forming. Indeed, the well-known expression given by the Swift' 52 diffuse necking approach in rigid-plasticity has been shown to be a natural outcome of the bifurcation analysis.

In the same way, Considère's criterion in classical elasto-plasticity has been given full justification within the bifurcation theory, whereby its proper extension to multiaxial loading conditions should be undertaken within the same theory of bifurcation. Indeed, this theoretical approach not only provides a sound foundation to the MFC for some particular constitutive equations, but also proves to be applicable and reliable in more general situations, while the MFC approach is not.

\section{Appendix A. Practical Formulas for a Set of Common Isotropic Hardening Models}

For completeness and practical applications, we provide in this appendix, the expressions of the critical equivalent strain and hardening moduli that are necessary for the evaluation of the critical strain and stress states. To this end, four isotropic hardening models are selected, which are widely used in the context of sheet metal forming. 
Table A.1. Critical equivalent strain and hardening modulus corresponding to rigid-plastic materials for different hardening models and three typical loading paths.

\begin{tabular}{lcccc}
\hline & & \multicolumn{3}{c}{ Loading path } \\
\cline { 3 - 5 } Hardening model & Critical state & UT & PT & EBE \\
\cline { 3 - 5 } & $\bar{\varepsilon}_{c}^{p}$ & $1-\frac{\sigma_{y}}{k}$ & $\frac{2}{\sqrt{3}}-\frac{\sigma_{y}}{k}$ & $2-\frac{\sigma_{y}}{k}$ \\
Linear hardening & $h^{c}$ & $k$ & $k$ & $k$ \\
Hollomon & $\bar{\varepsilon}_{c}^{p}$ & $n$ & $\frac{2}{\sqrt{3}} n$ & $2 n$ \\
$Y=k\left(\bar{\varepsilon}^{p}\right)^{n}$ & $h^{c}$ & $k n^{n}$ & $k n^{n}\left(\frac{2}{\sqrt{3}}\right)^{n-1}$ & $k n(2 n)^{n-1}$ \\
Swift & $\bar{\varepsilon}_{c}^{p}$ & $n-\varepsilon_{0}$ & $\frac{2}{\sqrt{3}} n-\varepsilon_{0}$ & $2 n-\varepsilon_{0}$ \\
$Y=k\left(\varepsilon_{0}+\bar{\varepsilon}^{p}\right)^{n}$ & $h^{c}$ & $k n^{n}$ & $k n^{n}\left(\frac{2}{\sqrt{3}}\right)^{n-1}$ & $k n(2 n)^{n-1}$ \\
Voce & $\bar{\varepsilon}_{c}^{p}$ & $\frac{1}{c} \ln \left(\frac{Q(c+1)}{\sigma_{y}+Q}\right)$ & $\frac{1}{c} \ln \left(\frac{Q(2 c / \sqrt{3}+1)}{\sigma_{y}+Q}\right)$ & $\frac{1}{c} \ln \left(\frac{Q(2 c+1)}{\sigma_{y}+Q}\right)$ \\
$Y=\sigma_{y}+Q\left(1-e^{-c \bar{\varepsilon}^{p}}\right)$ & $h^{c}$ & $\frac{c\left(\sigma_{y}+Q\right)}{(c+1)}$ & $\frac{c \sqrt{3}\left(\sigma_{y}+Q\right)}{(2 c+\sqrt{3})}$ & $\frac{c\left(\sigma_{y}+Q\right)}{(2 c+1)}$ \\
\hline
\end{tabular}

Note that the above critical values of hardening moduli and equivalent strains are also valid in elasto-plasticity when $h / E \ll 1$, for both the bifurcation approach and the MFC. Therefore, the results of Table A.1 can be used to completely specify the elasto-plastic critical states investigated in Sec. 4 , provided that $h / E \ll 1$, for both bifurcation analysis (in combination with Table 3) and MFC (along with Table 4).

Table A.2. Critical strains corresponding to bifurcation for rigid-plastic materials (or elasto-plastic materials with $h / E \ll 1$ ) for a set of popular hardening models.

\begin{tabular}{lcc}
\hline Hardening law & Critical equivalent strain $\bar{\varepsilon}_{c}^{p}$ & Critical major strain $\varepsilon_{1}^{c}$ \\
\hline Linear & $\frac{4\left(\alpha^{2}-\alpha+1\right)^{3 / 2}}{(1+\alpha)\left(4 \alpha^{2}-7 \alpha+4\right)}-\frac{\sigma_{y}}{k}$ & $\frac{2-\alpha}{2 \sqrt{\alpha^{2}-\alpha+1}}\left(\frac{4\left(\alpha^{2}-\alpha+1\right)^{3 / 2}}{(1+\alpha)\left(4 \alpha^{2}-7 \alpha+4\right)}-\frac{\sigma_{y}}{k}\right)$ \\
Hollomon & $\frac{4\left(\alpha^{2}-\alpha+1\right)^{3 / 2} n}{(1+\alpha)\left(4 \alpha^{2}-7 \alpha+4\right)}$ & $\frac{2\left(\alpha^{2}-\alpha+1\right)(2-\alpha) n}{(1+\alpha)\left(4 \alpha^{2}-7 \alpha+4\right)}$ \\
Swift & $\frac{4\left(\alpha^{2}-\alpha+1\right)^{3 / 2} n}{(1+\alpha)\left(4 \alpha^{2}-7 \alpha+4\right)}-\varepsilon_{0}$ & $\frac{2-\alpha}{2 \sqrt{\alpha^{2}-\alpha+1}}\left(\frac{4\left(\alpha^{2}-\alpha+1\right)^{3 / 2} n}{(1+\alpha)\left(4 \alpha^{2}-7 \alpha+4\right)}-\varepsilon_{0}\right)$ \\
Voce & $\frac{1}{c} \ln \left(\left(\frac{Q}{\sigma_{y}+Q}\right)\left(\frac{c}{g_{1}(\alpha)}+1\right)\right)$ & $\frac{2-\alpha}{2 c \sqrt{\alpha^{2}-\alpha+1}} \ln \left(\left(\frac{Q}{\sigma_{y}+Q}\right)\left(\frac{c}{g_{1}(\alpha)}+1\right)\right)$ \\
\hline
\end{tabular}

Table A.3. Critical strains corresponding to MFC for rigid-plastic materials (or elasto-plastic materials with $h / E \ll 1$ ) for a set of popular hardening models.

\begin{tabular}{lcc}
\hline Hardening law & Critical equivalent strain $\bar{\varepsilon}_{c}^{p}$ & Critical major strain $\varepsilon_{1}^{c}$ \\
\hline Linear & $\frac{2 \sqrt{\alpha^{2}-\alpha+1}}{(2-\alpha)}-\frac{\sigma_{y}}{k}$ & $1-\frac{\sigma_{y}}{2 k} \frac{(2-\alpha)}{\sqrt{\alpha^{2}-\alpha+1}}$ \\
Hollomon & $\frac{2 \sqrt{\alpha^{2}-\alpha+1}}{(2-\alpha)} n$ & $n$ \\
Swift & $\frac{2 \sqrt{\alpha^{2}-\alpha+1}}{(2-\alpha)} n-\varepsilon_{0}$ & $n-\frac{(2-\alpha)}{2 \sqrt{\alpha^{2}-\alpha+1}} \varepsilon_{0}$ \\
Voce & $\frac{1}{c} \ln \left(\left(\frac{Q}{\sigma_{y}+Q}\right)\left(\frac{c}{g_{2}(\alpha)}+1\right)\right)$ & $\frac{2-\alpha}{2 c \sqrt{\alpha^{2}-\alpha+1}} \ln \left(\left(\frac{Q}{\sigma_{y}+Q}\right)\left(\frac{c}{g_{2}(\alpha)}+1\right)\right)$ \\
\hline
\end{tabular}


For the other loading paths, the critical strains in rigid-plasticity, which are also valid in elasto-plasticity as long as $h / E \ll 1$, are given in Table A.2 for the bifurcation analysis, and in Table A.3 for the MFC (using the set of hardening models described above).

In Table A.2, $g_{1}(\alpha)=(1+\alpha)\left(\alpha^{2}-7 / 4 \alpha+1\right) /\left(\alpha^{2}-\alpha+1\right)^{3 / 2}$, while in Table A.3, $g_{2}(\alpha)=(1-\alpha / 2) / \sqrt{\alpha^{2}-\alpha+1}$. In both the tables, the critical minor strain is simply determined by $\varepsilon_{2}^{c}=\beta \varepsilon_{1}^{c}=(2 \alpha-1) /(2-\alpha) \varepsilon_{1}^{c}$.

\section{References}

Abed-Meraim, F. [1999] "Sufficient conditions for stability of viscous solids," Comptes Rendus de l'Académie des Sciences - Series IIb - Mechanics-Physics-Astronomy 327, 25-31.

Abed-Meraim, F. and Nguyen, Q. S. [2007] "A quasi-static stability analysis for Biot's equation and standard dissipative systems," European Journal of Mechanics A/Solids 26, 383-393.

Barbier, G., Benallal, A. and Cano, V. [1998] "Relation théorique entre la méthode de perturbation linéaire et l'analyse de bifurcation pour la prédiction de la localisation des deformations," Comptes Rendus de l'Académie des Sciences — Series IIb — Mechanics-Physics-Astronomy 326, 153-158.

Benallal, A. [2008] "A note on ill-posedness for rate-dependent problems and its relation to the rate-independent case," Computational Mechanics 42, 261-269.

Bigoni, D. and Hueckel, T. [1991] "Uniqueness and localization — associative and nonassociative elastoplasticity," International Journal of Solids Structures 28, 197-213.

Budiansky, B. [1974] "Theory of buckling and post-buckling behaviour of elastic structures," Advances in Applied Mechanics 14, 1-65.

Considère, A. [1885] "Mémoire sur l'emploi du fer et de l'acier dans les constructions," Annales des Ponts et Chaussées 9, 574-775.

Doghri, I. and Billardon, R. [1995] "Investigation of localization due to damage in elastoplastic materials," Mechanics of Materials 19, 129-149.

Drucker, D. C. [1956] "On uniqueness in the theory of plasticity," Quarterly of Applied Mathematics 14, 35-42.

Franz, G., Abed-Meraim, F., Ben Zineb, T., Lemoine, X. and Berveiller, M. [2009] "Strain localization analysis using a multiscale model," Computational Materials Science 45, $768-773$.

Franz, G., Abed-Meraim, F., Ben Zineb, T., Lemoine, X. and Berveiller, M. [2011] "Impact of intragranular microstructure development on ductility limits of multiphase steels," Materials Science and Engineering A 528, 3777-3785.

Franz, G., Abed-Meraim, F. and Berveiller, M. [2013] "Strain localization analysis for single crystals and polycrystals: Towards microstructure-ductility linkage," International Journal of Plasticity 48, 1-33.

Goodwin, G. M. [1968] "Application of strain analysis to sheet metal forming problems in the press shop," La Metallurgica Italiana 8, 767-774.

Gulshan Taj, M. N. A. and Chakrabarti, A. [2013] "Buckling analysis of functionally graded skew plates: An efficient finite element approach," International Journal of Applied Mechanics 5, 1350041.

Habbad, M. [1994] "Instabilités plastiques en élasto-plasticité anisotrope et grandes deformations," Ph.D. thesis, Ecole Centrale de Lyon, France. 
Haddag, B., Abed-Meraim, F. and Balan, T. [2009] "Strain localization analysis using a large deformation anisotropic elastic-plastic model coupled with damage," International Journal of Plasticity 25, 1970-1996.

Hill, R. [1952] "On discontinuous plastic states, with special reference to localized necking in thin sheets," Journal of the Mechanics and Physics of Solids 1, 19-30.

Hill, R. [1958] "A general theory of uniqueness and stability in elastic-plastic solids," Journal of the Mechanics and Physics of Solids 6, 236-249.

Hora, P., Tong, L. and Reissner, J. [1996] "A prediction method of ductile sheet metal failure in FE simulation," Proceedings of Numisheet 1996, Dearborn, Michigan, USA, pp. $252-256$.

Hutchinson, J. W. [1974] "Plastic buckling," Advances in Applied Mechanics 14, 67-144.

Hutchinson, J. W., Neale, K. W. [1978] "Sheet Necking - II. Time-independent behavior. In: D. P. Koistinen and N. M. Wang (Eds.), Mechanics of sheet metal forming. New York: Plenum Press, pp. 127-153.

Keeler, S. P. [1965] "Determination of forming limits in automotive stampings," Sheet Metal Industries 42, 683-691.

Koiter, W. T. [1945] "On the stability of elastic equilibrium," Ph.D. thesis, Delft. English translation NASA Techn. Trans. F10, 1967.

Liu, Z. S., Swaddiwudhipong, S., Cui, F. S., Hong, W., Suo, Z. and Zhang, Y. W. [2011] "Analytical solutions of polymeric gel structures under buckling and wrinkle," International Journal of Applied Mechanics 3, 235-257.

Lyapunov, A. [1892] The General Problem of Stability of Motion, English Translation (1992) (Taylor and Francis, London).

Mansouri, L. Z., Chalal, H. and Abed-Meraim, F. [2014] "Ductility limit prediction using a GTN damage model coupled with localization bifurcation analysis," Mechanics of Materials 76, 64-92.

Marciniak, Z. and Kuczyński, K. [1967] "Limit strains in the processes of stretch-forming sheet metal," International Journal of Mechanical Sciences 9, 613-620.

Mattiasson, K., Sigvant, M. and Larson, M. [2006] "Methods for forming limit prediction in ductile metal sheets," Proceedings of IDDRG 2006, Porto, Portugal, pp. 1-9.

Mojahedi, M., Ahmadian, M. T. and Firoozbakhsh, K. [2013] "Dynamic pull-in instability and vibration analysis of a nonlinear microcantilever gyroscope under step voltage considering squeeze film damping," International Journal of Applied Mechanics 5, 1350032 .

Molinari, A. and Clifton, R. [1987] "Analytical characterization of shear localization in thermo-visco-plastic solids," Journal of Applied Mechanics 54, 806-812.

Neilsen, M. K. and Schreyer, H. L. [1993] "Bifurcations in elastic-plastic materials," International Journal of Solids and Structures 30, 521-544.

Rice, J. R. [1976] "The localization of plastic deformation," Proceedings of the 14 th International Congress on Theoretical and Applied Mechanics, North-Holland Publishing Co., Delft, Netherlands, pp. 207-220.

Rudnicki, J. W. and Rice, J. R. [1975] "Conditions for the localization of deformation in pressure-sensitive dilatant materials," Journal of the Mechanics and Physics of Solids 23, 371-394.

Swift, H. W. [1952] "Plastic instability under plane stress," Journal of the Mechanics and Physics of Solids 1, 1-18.

Timoshenko, S. P. and Gere, J. M. [1961] Theory of Elastic Stability (McGraw-Hill, New York).

Toh, W., Liu, Z., Ng, T. Y. and Hong, W. [2013] "Inhomogeneous large deformation kinetics of polymeric gels," International Journal of Applied Mechanics 5, 1350001. 
Valanis, K. C. [1989] "Banding and stability in plastic materials," Acta Mechanica 79, $113-141$.

Van der Sluis, O., Hsu, Y. Y., Timmermans, P. H. M., Gonzalez, M. and Hoefnagels, J. P. M. [2011] "Stretching-induced interconnect delamination in stretchable electronic circuits," Journal of Physics D: Applied Physics 44, 034008. 